\title{
VGF Expression by Monocytes in Patients with Alzheimer's Disease and Vascular Dementia
}

\author{
Stefan Busse ${ }^{1} \oplus$, Eva Meyer ${ }^{1}$, Henrik Dobrowolny ${ }^{1}$, Christian Mawrin ${ }^{2}$, Roland Hartig ${ }^{3}$, \\ Bernhard Bogerts ${ }^{1}$, and Mandy Busse ${ }^{1,4}$ \\ ${ }^{1}$ Department of Psychiatry, University of Magdeburg, Germany \\ ${ }^{2}$ Department of Neuropathology, University of Magdeburg, Germany \\ ${ }^{3}$ Institute for Molecular and Clinical Immunology, University of Magdeburg, Germany \\ ${ }^{4}$ Experimental Obstetrics and Gynecology, University of Magdeburg, Germany
}

Abstract: Because its secretion is changed in cerebrospinal fluid and peripheral blood, the neuronal polypeptide VGF (nonacronymic) has been discussed as a biomarker for neuropsychiatric disorders. We have shown an enhanced VGF expression by T-cells from Alzheimer's disease (AD) patients. In this study, we investigated the VGF expression by peripheral monocytes in 38 AD patients, 5 patients with vascular dementia (VD), and 20 neuropsychiatrically healthy individuals using flow cytometry. We determined an enhanced number of VGF-expressing monocytes in VD patients compared to AD patients. VGF+CD14+ monocytes were not correlated with age, body mass index, Mini-Mental State Examination (MMSE), or Q albumin. These preliminary data support findings indicating that VGF might play a role as a peripheral biomarker in VD.

Keywords: Alzheimer's disease, vascular dementia, monocytes, VGF, biomarker

\section{Introduction}

Age-related diseases are becoming of great importance, mainly because of increases in population aging and growth (Collaborators, 2019). Between 1990 and 2016, the number of patients with dementia worldwide increased from 20.2 million to 43.8 million. After cardiovascular disorders and cancer, the different forms of dementia are the most frequent diseases in patients over 65 years of age.

$60-70 \%$ of demented persons suffer from Alzheimer's disease (AD) (Ferri et al., 2005). Clinically, this neurodegenerative disease is characterized by a loss of memory and cognitive functions, while neuropathologically there is a loss of neurons, white matter, and synapses primarily in the hippocampus and the temporal cortex. These neuropathological changes include extracellular amyloid plaques and intracellular neurofibrillary tangles, which might result in neuroinflammation (Calderon-Garciduenas \& Duyckaerts, 2017). Microvascular pathologies next to multiple, large volume or lacunar brain infarcts are one of the hallmarks of vascular dementia (VD), the second most common cause of dementia. VD and AD both have several risk factors such as hypertension, diabetes mellitus, and hypercholesterolemia, which are responsible for damage to the blood-brain barrier. Additionally, microvascular pathologies are observed in $\mathrm{AD}$ (Calabro et al., 2021). If this barrier shows dysfunction, the passage of hematopoietic inflammatory cells into the central nervous system (CNS) seems possible.

Microglia are the brain's tissue macrophage and belong to the innate immune cells of the CNS. They account for $10-15 \%$ of the adult glial cells in the brain (Nayak et al., 2014). This cell population exhibits an important function since they protect from infections and maintain brain homeostasis (Mandrekar-Colucci \& Landreth, 2010). It was shown that microglia take up and dispose of cellular debris, and that they accumulate at the amyloid plaques.

Neuroinflammation is considered to play an important role in $\mathrm{AD}$ as well as VD.

Inflammatory processes result in the activation of microglia, which are present in the amyloid plaques (Lue et al., 2010). Cytokines like IL-1beta and IL-6, which initiate similar processes in microglia as well as in the periphery are upregulated in $\mathrm{AD}$ compared to nondemented elderly persons (Magalhaes et al., 2018; Ng et al., 2018; RiveraEscalera et al., 2014).

We determined no age-related alterations in the peripheral frequency of CD14+ monocytes and no differences in 
Table 1. Demographic data of study cohort

\begin{tabular}{|c|c|c|c|c|c|c|}
\hline Characteristics & Controls & $A D$ & $p$ (vs. control) & VD & $p$ (vs. control) & $p$ (vs. AD) \\
\hline Total $(n)$ & 20 & 38 & & 5 & & \\
\hline Age (years; median) & 74.50 & 80.00 & .008 & 80.00 & 0.246 & 1.000 \\
\hline Sex (female/male) & $14 / 6$ & $21 / 17$ & 1.000 & $3 / 2$ & 1.000 & 1.000 \\
\hline BMI (median) & 25.2 & 24.67 & 1.000 & 24.67 & 1.000 & 1.000 \\
\hline MMSE (median) & 29.00 & 18.50 & $<.001$ & 20.00 & 0.003 & 1.000 \\
\hline p tau (median) & NA & 83.00 & NA & 42.00 & NA & .007 \\
\hline Amyloid- $\beta$ 1-42 (median) & NA & 444.5 & NA & 501.0 & NA & .248 \\
\hline Amyloid- $\beta$ ratio (median) & NA & 0.60 & NA & 0.70 & NA & .163 \\
\hline Q albumin (median) & NA & 7.400 & NA & 5.200 & NA & 1.000 \\
\hline
\end{tabular}

Note. NA = not available.

blood monocytes between $\mathrm{AD}$ patients and age-matched neuropsychiatric healthy persons, albeit a decreased number of monocytes in VD patients (Busse et al., 2017; Busse, Steiner, Alter, et al., 2015). We further identified peripheral monocytes that expressed quinolinic acid, a neurotoxic agent (Busse et al., 2018). Therefore, we addressed the question of whether peripheral monocytes express further molecules that might be of particular interest in neurodegenerative disorders.

VFG is such a molecule that might play a role in dementia (Beckmann et al., 2020; Cocco et al., 2010; El Gaamouch et al., 2020; Llano et al., 2019). VGF is widely expressed in the neurons in the brain, especially the hippocampus (Lewis et al., 2015), the brain region that is usually most affected in $\mathrm{AD}$ (Jaroudi et al., 2017). VGF plays an important role by improving plasticity, neurogenesis, and energy homeostasis (Behnke et al., 2017; Busse et al., 2012; Sadahiro et al., 2015; Saderi et al., 2014; Thakker-Varia et al., 2014). A disturbance in energy balance is present in diabetes mellitus, which is considered a risk factor for the development of AD (Baglietto-Vargas et al., 2016; Pugazhenthi et al., 2017). Recently, we showed that VGF is expressed by a higher percentage of peripheral CD3+ $\mathrm{T}$-cells from $\mathrm{AD}$ patients than in age-matched neuropsychiatric healthy volunteers (Busse, Steiner, Glorius, et al., 2015). In this study, we determined the expression of VGF in monocytes of patients with $\mathrm{AD}$ and VD compared with that of nondemented persons.

\section{Materials and Methods}

\section{Study Cohort}

The study was performed in accordance with German regulations, the Declaration of Helsinki, and the guidelines of the local institutional review board. Written consent was obtained from all patients and healthy persons. We collected $18 \mathrm{ml}$ blood from $38 \mathrm{AD}$ patients, $5 \mathrm{VD}$ patients, and 20 sex-matched subjects without neuropsychiatric disorders (the demographic features are summarized in Table 1). A diagnosis of dementia was based on a combination of the patient's history, an examination including neuropsychological testing, and further diagnostics (magnetic resonance imaging and cerebrospinal fluid). A patient's history was characterized by progressive deterioration in cognitive abilities. The Mini-Mental State Examination (MMSE) and Consortium to Establish a Registry for Alzheimer's Disease (CERAD) were performed to evaluate the cognitive function of the patients. Patients with $\mathrm{AD}$ showed a hippocampal and/or mesiotemporal atrophy and characteristic changes in cerebrospinal fluid (CSF) values ( $\mathrm{p}$ tau $>50 \mathrm{pg} / \mathrm{ml}$, total tau $>350 \mathrm{pg} / \mathrm{ml}, \mathrm{A} \beta 1-42<0.8$ ). Routine blood analysis including differential blood cell count, levels of C-reactive protein, glucose, lipids, liver enzymes, and thyroid hormones were also performed. Persons with a history of autoimmune disorders, immunomodulating treatment, cancer, chronic terminal disease, severe cardiovascular disorder, substance abuse, or severe trauma were excluded from the study.

\section{Preparation of Peripheral Blood Monocyte Cells (PBMC)}

Peripheral blood mononuclear cells (PBMCs) were separated by standard density gradient centrifugation (Ficoll Paque; Biochrom AG, Berlin) for $20 \mathrm{~min}, 375 \mathrm{x} \mathrm{g}$, at room temperature, from freshly drawn blood collected in lithium heparin-treated tubes (BD Vacutainer; BD Biosciences, San Jose, CA, USA), which was diluted 1:1 with phosphatebuffered saline (PBS). The cell ring between Ficoll Paque and PBS was harvested, and the cell suspension was centrifuged and washed twice in PBS. Cells were suspended in staining buffer (PBS w/0.5\% BSA), and the cell number was determined. 
(A)

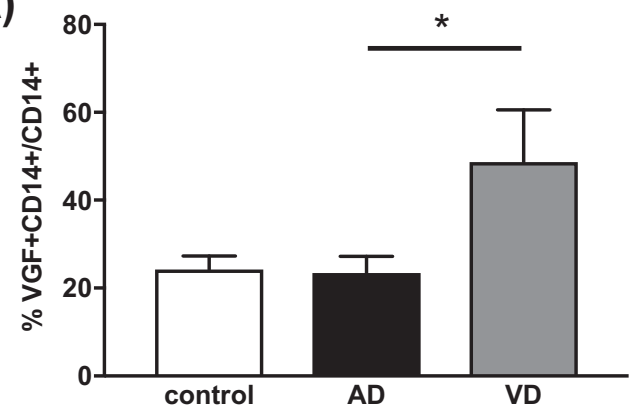

(B)

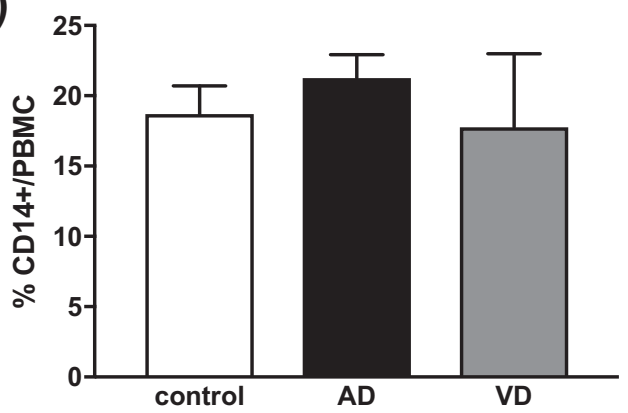

Figure 1. VGF+CD14+ monocytes are increased in VD patients. The number of VGF-expressing monocytes (A) and the number of monocytes (B) were determined in peripheral blood of 38 AD and 5 VD patients and 20 neuropsychiatric healthy control persons. ${ }^{\star} p<.05$.

\section{Flow Cytometry}

Isolated PBMCs were washed once and then incubated with fluoresce-labeled antibodies for $20 \mathrm{~min}$ at $4{ }^{\circ} \mathrm{C}$ in staining buffer (1\% BSA in PBS). Antibodies (Abs) used in this study included reagents specific for CD14 (TÜK4) from Miltenyi Biotec (Bergisch Gladbach, Germany); VGF (D-20) and the detection antibody, donkey anti-goat IgG FITC, from Santa Cruz (Dallas, TX, USA). Data were collected on LSRFortessa and analyzed using FACS DIVA software (BD Biosciences, Mountain View, CA, USA). The frequency of cells was shown by using the biexponential transformation function for complete data visualization.

\section{Statistical Analysis}

Statistics were performed using SPSS 26 and GraphPad Prism 7. Shapiro-Wilk tests were performed to test for normal distribution. In dependence of the obtained results, the differences between the groups were calculated by one-way ANOVA or Kruskal-Wallis H-test, followed by Bonferroni posthoc tests. Gender differences were calculated using the chi-square test. Correlation analyses were performed using Spearman analysis. Significance was defined as $p<.05$.

\section{Results}

\section{The Expression of VGF in Monocytes Is Enhanced in VD Patients}

In the elderly neuropsychiatrically healthy control group, the median frequency of CD14+ monocytes in the PBMC fraction was $18.5 \%$ (Table 1). This number decreased in VD patients to $13.2 \%$, whereas in AD patients it was nearly unchanged (19.3\%).

In the group of neuropsychiatrically healthy persons, on average $21.3 \%$ of monocytes expressed VGF. At the time when $\mathrm{AD}$ was diagnosed, the percentage of monocytes expressing VGF was $15 \%$, the frequency of VGF+CD14+ cells from VD patients enhanced to $36.5 \%$ ( $p=.034$; see Figure 1).

\section{Correlation of VGF Expression by Monocytes with Age and $Q$ albumin}

Since we determined an age-dependent increase in the frequency of VGF-expressing T-cells in healthy individuals (Busse et al., 2014) and with enhancing BMI values (Busse et al., 2014), we addressed the question of whether age, BMI, MMSE, and Q albumin also influenced the expression of VGF by CD14+ monocytes.

First, because of age differences between the control group and the demented groups, we found that age negatively influenced the MMSE values. Moreover, age also influenced $Q$ albumin as well as the $A \beta$ ratio, especially in $\mathrm{AD}$, especially in female patients (Table 2).

However, no significant correlations were determined between age (Figure 2A), BMI (Figure 2B), MMSE (Figure 2C), or $\mathrm{Q}$ albumin (Figure 2D) and the frequency of VGF-expressing CD14+ monocytes, neither in the total study cohort (Figure 2) nor in the individual control, AD, and VD groups (data not shown).

\section{Discussion}

The present study found that monocytes obtained by VD patients expressed more VGF than monocytes from $\mathrm{AD}$ patients or elderly neuropsychiatric healthy persons, while the frequency of peripheral CD14+ monocytes was not changed.

Our results are in line with our previous finding showing no differences in the percentage of CD14+ cells from $\mathrm{AD}$ patients at the time of diagnosis compared to the control group (Busse, Steiner, Alter, et al., 2015), while in VD 
Table 2. Influence of age

\begin{tabular}{|c|c|c|c|c|c|c|}
\hline \multirow[b]{2}{*}{ Variable } & \multirow[b]{2}{*}{ Total age } & \multicolumn{3}{|c|}{ Group } & \multicolumn{2}{|c|}{ Gender } \\
\hline & & Age controls & Age AD & Age VD & Female & Male \\
\hline \multicolumn{7}{|l|}{ BMI } \\
\hline Rho & -0.375 & -1.000 & -0.239 & -1.000 & -0.266 & -0.490 \\
\hline$p$ & $0.034^{*}$ & & 0.230 & $<0.001 * * *$ & 0.302 & 0.064 \\
\hline \multicolumn{7}{|l|}{ MMSE } \\
\hline Rho & -0.310 & 0.075 & 0.001 & -0.400 & -0.276 & 0.011 \\
\hline$p$ & $0.024^{*}$ & 0.807 & 0.997 & 0.600 & 0.133 & 0.966 \\
\hline \multicolumn{7}{|c|}{ Amyloid-beta 1-42 } \\
\hline Rho & -0.027 & NA & -0.039 & -0.100 & -0.024 & 0.011 \\
\hline$p$ & 0.867 & & 0.821 & 0.873 & 0.916 & 0.966 \\
\hline \multicolumn{7}{|c|}{ Phospho tau } \\
\hline Rho & 0.036 & NA & 0.106 & -0.500 & 0.196 & -0.062 \\
\hline$p$ & 0.819 & & 0.533 & 0.391 & 0.370 & 0.800 \\
\hline \multicolumn{7}{|c|}{ Amyloid-beta ratio } \\
\hline Rho & -0.370 & NA & -0.488 & 0.462 & -0.575 & -0.013 \\
\hline$p$ & $0.016^{*}$ & & $0.002^{\star *}$ & 0.434 & $0.004^{* *}$ & 0.957 \\
\hline \multicolumn{7}{|c|}{ \%VGF+CD14+/+CD14+ } \\
\hline Rho & -0.008 & 0.136 & -0.026 & 0.800 & -0.028 & 0.068 \\
\hline$p$ & 0.949 & 0.567 & 0.878 & 0.104 & 0.868 & 0.742 \\
\hline \multicolumn{7}{|l|}{ \%CD14+ } \\
\hline Rho & 0.014 & -0.003 & -0.181 & -0.200 & 0.122 & -0.024 \\
\hline$p$ & 0.916 & 0.990 & 0.278 & 0.747 & 0.472 & 0.906 \\
\hline
\end{tabular}

(A)

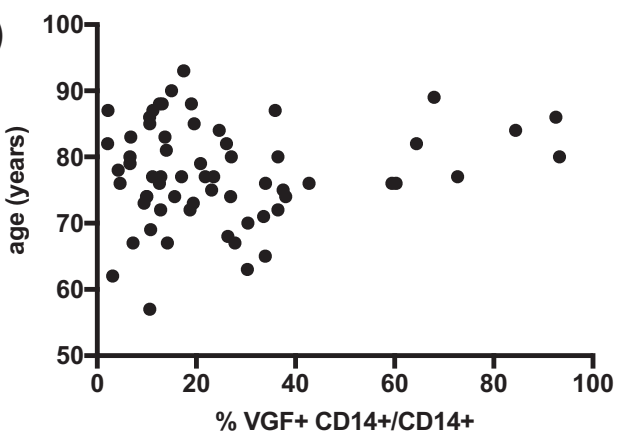

(C)

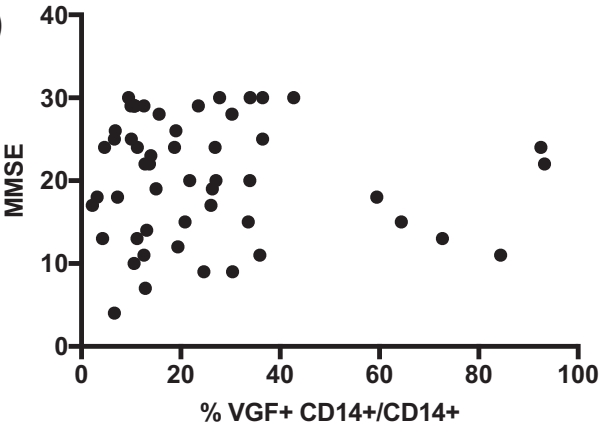

(B)

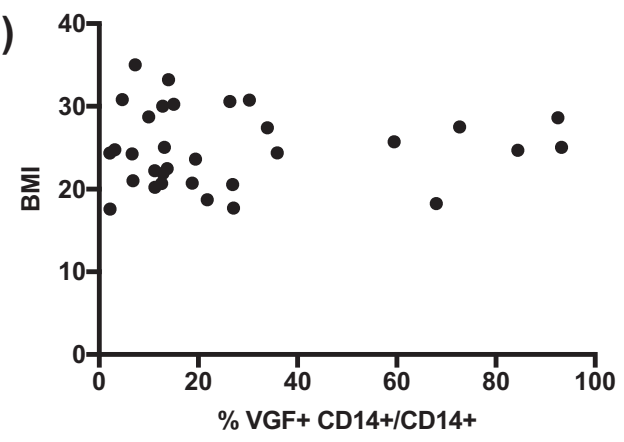

(D)

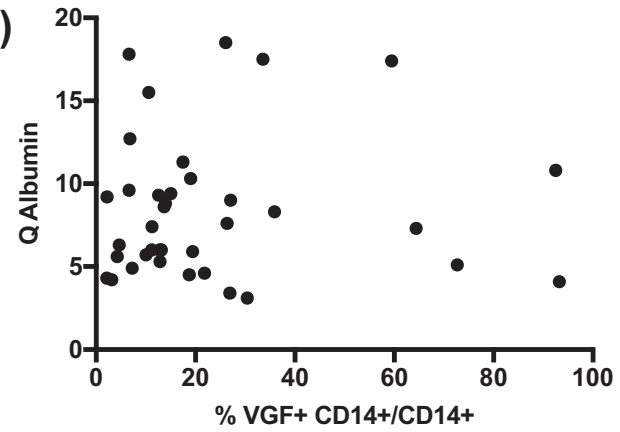

Figure 2. Correlations between age, BMI, MMSE, and $Q$ albumin and the frequency of VGF-expressing CD14+ monocytes. No significant correlations were determined between age (A), BMI (B), MMSE (C), and Q albumin (D) and the frequency of VGF-expressing CD14+ monocytes in the total study cohort. 
patients the number of CD14+ monocytes was decreased (Busse et al., 2017).

Although changes in blood cell profile of the AD and VD types of dementia are not yet fully understood, some reports show an altered peripheral blood cells profile. Chen et al. (2017) discovered a decreased population of lymphocytes and basophils in $\mathrm{AD}$ patients compared with controls, but an unaltered level of monocytes. In contrast, Lunnon et al. (2012) revealed an increase in the number of monocytes in the blood of patients with AD. In this study, we show that VGF is expressed by a higher percentage of peripheral $\mathrm{CD} 14+$ monocytes from VD patients than in age-matched neuropsychiatric healthy volunteers and in $\mathrm{AD}$ patients.

VD is characterized by strokes and vascular damage. This also often means a lack of the blood-brain barrier (BBB) (Ueno et al., 2016). As a result of the disturbed BBB, monocytes can immigrate into the brain. In their review, Han et al. (2020) described that, following an acute ischemic stroke, peripheral monocytes infiltrate into the lesion site within $24 \mathrm{~h}$ and peak at 3 to 7 days. Increasing evidence indicates that alterations of the cerebral microcirculation may also play a role in $\mathrm{AD}$ (Park et al., 2017). Because a dysfunctional $\mathrm{BBB}$ is also often associated with $\mathrm{AD}$, there are great similarities between $\mathrm{AD}$ and VD (Burgmans et al., 2013). In contrast to patients with VD, however, we did not find any increased expression of VGF in monocytes in patients with $\mathrm{AD}$. A decisive neuropathological difference between $\mathrm{AD}$ and VD lies in the amyloid plaques surrounded by activated microglia.

We previously showed that the expression of VGF by peripheral T-cells is age-dependent (Busse et al., 2014). In our present study, however, we did not detect a correlation between age and VGF-expressing monocytes, although the control persons were younger than the demented patients. Since we did not include healthy persons from younger age brackets, we cannot draw any conclusion whether the VGF expression by monocytes changes with enhanced age in healthy persons.

Recently, it was shown that the expression of VGF is required by rapid-acting antidepressants that modulate $\mathrm{N}$ methyl-D-aspartate receptor (NMDA-R) signaling (Jiang et al., 2019). This is of particular interest since the NMDA-R is very important in the pathology of AD (Foster et al., 2017). In this context, it is interesting to note that serum VGF levels were significantly lower in patients with major depressive disorder than in bipolar depression (BD) patients, but higher in $\mathrm{BD}$ patients compared to healthy control individuals. No correlation was found between serum VGF levels and any data of persons (Chen et al., 2019).

The frequency of VGF-expressing T-cells increased with enhanced BMI (Busse et al., 2014). On the other hand, the VGF peptides NAPPE and TLQP were significantly reduced in the plasma of obese persons, defined by BMI (D'Amato et al., 2015). The frequency of VGF-expressing CD14+ monocytes was not altered by BMI.

It was shown that VGF levels in CSF were lower in Lewy body dementia (LBD) than in AD patients or controls and are associated with a stronger cognitive decline. VGF in LBD was positively correlated with tau, but not A $\beta 1-42$ in CSF (Van Steenoven et al., 2019). Another group found a decreased VGF level in CSF of AD patients (Sathe et al., 2019). However, the frequency of VGF+ monocytes was not correlated with the mental state of the study cohort nor with the cognitive decline in $\mathrm{AD}$ and VD patients. It remains to be clarified whether the plasma or serum level of VGF is altered in AD and VD as well, and whether there is a correlation between CSF and plasma/serum VGF level.

The present study has a certain limitation that must be considered: We analyzed the VGF expression in $38 \mathrm{AD}$ patients, but only in 5 patients with VD. Further studies should confirm these preliminary data.

Taken together, VGF is expressed by monocytes, but while the expression level is not altered between $\mathrm{AD}$ patients and nondemented controls, it is increased in VD. Since it is not correlated with age, BMI, MMSE, or Q albu$\mathrm{min}$, it might be rather associated with a VD-specific characteristic.

\section{References}

Baglietto-Vargas, D., Shi, J., Yaeger, D. M., Ager, R., \& LaFerla, F. M. (2016). Diabetes and Alzheimer's disease crosstalk. Neuroscience and Biobehavioral Reviews, 64, 272-287. https://doi.org/10.1016/j.neubiorev.2016.03.005

Beckmann, N. D., Lin, W. J., Wang, M., Cohain, A. T., Charney, A. W., Wang, P., Ma, W., Wang, Y. C., Jiang, C., Audrain, M., Comella, P. H., Fakira, A. K., Hariharan, S. P., Belbin, G. M., Girdhar, K., Levey, A. I., Seyfried, N. T., Dammer, E. B., Duong, D., ... Schadt, E. E. (2020). Multiscale causal networks identify VGF as a key regulator of Alzheimer's disease. Nature Communications, 11(1), Article 3942. https://doi.org/10.1038/ s41467-020-17405-z

Behnke, J., Cheedalla, A., Bhatt, V., Bhat, M., Teng, S., Palmieri, A., Windon, C. C., Thakker-Varia, S., \& Alder, J. (2017). Neuropeptide VGF promotes maturation of hippocampal dendrites that is reduced by single nucleotide polymorphisms. International Journal of Molecular Science, 18(3), Article 612. https://doi. org/10.3390/ijms18030612

Burgmans, S., van de Haar, H. J., Verhey, F. R., \& Backes, W. H. (2013). Amyloid-beta interacts with blood-brain barrier function in dementia: A systematic review. Journal of Alzheimer's Disease, 35(4), 859-873. https://doi.org/10.3233/JAD-122155

Busse, M., Hettler, V., Fischer, V., Mawrin, C., Hartig, R., Dobrowolny, H., Bogerts, B., Frodl, T., \& Busse, S. (2018). Increased quinolinic acid in peripheral mononuclear cells in Alzheimer's dementia. European Archives of Psychiatry and Clinical Neuroscience, 268(5), 493-500. https://doi.org/ $10.1007 / s 00406-017-0785-y$ 
Busse, M., Michler, E., von Hoff, F., Dobrowolny, H., Hartig, R., Frodl, T., \& Busse, S. (2017). Alterations in the peripheral immune system in dementia. Journal of Alzheimer's Disease, 58(4), 1303-1313. https://doi.org/10.3233/JAD-161304

Busse, S., Bernstein, H. G., Busse, M., Bielau, H., Brisch, R., Mawrin, C., Muller, S., Sarnyai, Z., Gos, T., Bogerts, B., \& Steiner, J. (2012). Reduced density of hypothalamic VGFimmunoreactive neurons in schizophrenia: A potential link to impaired growth factor signaling and energy homeostasis. European Archives of Psychiatry and Clinical Neuroscience, 262 (5), 365-374. https://doi.org/10.1007/s00406-011-0282-7

Busse, S., Steiner, J., Alter, J., Dobrowolny, H., Mawrin, C., Bogerts, B., Hartig, R., \& Busse, M. (2015). Expression of HLA-DR, CD80, and CD86 in healthy aging and Alzheimer's disease. Journal of Alzheimer's Disease, 47(1), 177-184. https://doi.org/10.3233/ JAD-150217

Busse, S., Steiner, J., Glorius, S., Dobrowolny, H., Greiner-Bohl, S., Mawrin, C., Bommhardt, U., Hartig, R., Bogerts, B., \& Busse, M. (2015). VGF expression by $T$ lymphocytes in patients with Alzheimer's disease. Oncotarget, 6(17), 14843-14851. https:// doi.org/10.18632/oncotarget.3569

Busse, S., Steiner, J., Micheel, J., Dobrowolny, H., Mawrin, C., Krause, T. J., Adamaszek, M., Bogerts, B., Bommhardt, U., Hartig, R., \& Busse, M. (2014). Age-related increase of VGFexpression in T lymphocytes. Aging (Albany NY), 6(6), 440-453. https://doi.org/10.18632/aging.100656

Calabro, M., Rinaldi, C., Santoro, G., \& Crisafulli, C. (2021). The biological pathways of Alzheimer disease: A review. AIMS Neuroscience, 8(1), 86-132. https://doi.org/10.3934/ Neuroscience. 2021005

Calderon-Garciduenas, A. L., \& Duyckaerts, C. (2017). Alzheimer disease. Handbook of Clinical Neurology, 145, 325-337. https:// doi.org/10.1016/B978-0-12-802395-2.00023-7

Chen, S., Jiang, H., Hou, Z., Yue, Y., Zhang, Y., Zhao, F., Xu, Z., Li, Y., Mou, X., Li, L., Wang, T., Zhao, J., Han, C., Sui, Y., Wang, M., Yang, Z., Lu, Y., Zhu, Y., Li, J., ... Yuan, Y. (2019). Higher serum VGF protein levels discriminate bipolar depression from major depressive disorder. Journal of Neuroscience Research, 97(5), 597-606. https://doi.org/10.1002/jnr.24377

Chen, S. H., Bu, X. L., Jin, W. S., Shen, L. L., Wang, J., Zhuang, Z. Q., Zhang, T., Zeng, F., Yao, X. Q., Zhou, H. D., \& Wang, Y. J. (2017). Altered peripheral profile of blood cells in Alzheimer disease: A hospital-based case-control study. Medicine (Baltimore), 96(21), e6843. https://doi.org/10.1097/MD.0000000000006843

Cocco, C., D’Amato, F., Noli, B., Ledda, A., Brancia, C., Bongioanni, P., \& Ferri, G. L. (2010). Distribution of VGF peptides in the human cortex and their selective changes in Parkinson's and Alzheimer's diseases. Journal of Anatomy, 217(6), 683-693. https://doi.org/10.1111/j.1469-7580.2010.01309.x

Collaborators, G. B. D. N. (2019). Global, regional, and national burden of neurological disorders, 1990-2016: A systematic analysis for the Global Burden of Disease Study 2016. Lancet Neurology, 18(5), 459-480. https://doi.org/10.1016/S14744422(18)30499-X

D’Amato, F., Noli, B., Angioni, L., Cossu, E., Incani, M., Messana, I., Manconi, B., Solinas, P., Isola, R., Mariotti, S., Ferri, G. L., \& Cocco, C. (2015). VGF peptide profiles in Type 2 diabetic patients' plasma and in obese mice. PLoS One, 10(11), e0142333. https://doi.org/10.1371/journal.pone.0142333

El Gaamouch, F., Audrain, M., Lin, W. J., Beckmann, N., Jiang, C., Hariharan, S., Heeger, P. S., Schadt, E. E., Gandy, S., Ehrlich, M. E., \& Salton, S. R. (2020). VGF-derived peptide TLQP-21 modulates microglial function through C3aR1 signaling pathways and reduces neuropathology in 5xFAD mice. Molecular
Neurodegeneration, 15(1), Article 4. https://doi.org/10.1186/ s13024-020-0357-x

Ferri, C. P., Prince, M., Brayne, C., Brodaty, H., Fratiglioni, L., Ganguli, M., Hall, K., Hasegawa, K., Hendrie, H., Huang, Y., Jorm, A., Mathers, C., Menezes, P. R., Rimmer, E., Scazufca, M., \& Alzheimer's Disease, I. (2005). Global prevalence of dementia: A Delphi consensus study. Lancet, 366(9503), 2112-2117. https://doi.org/10.1016/S0140-6736(05)67889-0

Foster, T. C., Kyritsopoulos, C., \& Kumar, A. (2017). Central role for NMDA receptors in redox mediated impairment of synaptic function during aging and Alzheimer's disease. Behavioural Brain Research, 322(Pt B), 223-232. https://doi.org/10.1016/j. bbr.2016.05.012

Han, D., Liu, H., \& Gao, Y. (2020). The role of peripheral monocytes and macrophages in ischemic stroke. Journal of Neurological Sciences, 41(12), 3589-3607. https://doi.org/10.1007/s10072020-04777-9

Jaroudi, W., Garami, J., Garrido, S., Hornberger, M., Keri, S., \& Moustafa, A. A. (2017). Factors underlying cognitive decline in old age and Alzheimer's disease: The role of the hippocampus. Reviews in the Neurosciences, 28(7), 705-714. https://doi.org/ 10.1515/revneuro-2016-0086

Jiang, C., Lin, W. J., \& Salton, S. R. (2019). Role of a VGF/BDNF/ TrkB autoregulatory feedback loop in rapid-acting antidepressant efficacy. Journal of Molecular Neuroscience, 68(3), 504509. https://doi.org/10.1007/s12031-018-1124-0

Lewis, J. E., Brameld, J. M., \& Jethwa, P. H. (2015). Neuroendocrine Role for VGF. Frontiers of Endocrinology (Lausanne), 6, Article 3. https://doi.org/10.3389/fendo.2015.00003

Llano, D. A., Devanarayan, P., Devanarayan, V., \& Alzheimer's Disease Neuroimaging, I. (2019). VGF in cerebrospinal fluid combined with conventional biomarkers enhances prediction of conversion from $\mathrm{MCl}$ to $\mathrm{AD}$. Alzheimer Disease and Associated Disordorders, 33(4), 307-314. https://doi.org/10.1097/ WAD.0000000000000328

Lue, L. F., Kuo, Y. M., Beach, T., \& Walker, D. G. (2010). Microglia activation and anti-inflammatory regulation in Alzheimer's disease. Molecular Neurobiology, 41(2-3), 115-128. https:// doi.org/10.1007/s12035-010-8106-8

Lunnon, K., Ibrahim, Z., Proitsi, P., Lourdusamy, A., Newhouse, S., Sattlecker, M., Furney, S., Saleem, M., Soininen, H., Kloszewska, I., Mecocci, P., Tsolaki, M., Vellas, B., Coppola, G., Geschwind, D., Simmons, A., Lovestone, S., Dobson, R., Hodges, A., \& AddNeuroMed, C. (2012). Mitochondrial dysfunction and immune activation are detectable in early Alzheimer's disease blood. Journal of Alzheimer's Disease, 30(3), 685-710. https:// doi.org/10.3233/JAD-2012-111592

Magalhaes, C. A., Ferreira, C. N., Loures, C. M. G., Fraga, V. G., Chaves, A. C., Oliveira, A. C. R., de Souza, L. C., Resende, E. P. F., Carmona, K. C., Guimaraes, H. C., Cintra, M. T. G., Lanna, I. N., Zauli, D. A. G., Bicalho, M. A., Carvalho, M. G., Sousa, L. P., Caramelli, P., \& Gomes, K. B. (2018). Leptin, hsCRP, TNF-alpha and IL-6 levels from normal aging to dementia: Relationship with cognitive and functional status. Journal of Clinical Neuroscience, 56, 150-155. https://doi.org/ 10.1016/j.jocn.2018.08.027

Mandrekar-Colucci, S., \& Landreth, G. E. (2010). Microglia and inflammation in Alzheimer's disease. CNS and Neurological Disorders - Drug Targets, 9(2), 156-167. https://doi.org/ 10.2174/187152710791012071

Nayak, D., Roth, T. L., \& McGavern, D. B. (2014). Microglia development and function. Annual Review of Immunology, 32, 367-402. https://doi.org/10.1146/annurev-immunol-032713120240 
Ng, A., Tam, W. W., Zhang, M. W., Ho, C. S., Husain, S. F., Mclntyre, R. S., \& Ho, R. C. (2018). IL-1beta, IL-6, TNF-alpha and CRP in elderly patients with depression or Alzheimer's disease: Systematic review and meta-analysis. Scientific Reports, 8(1), Article 12050. https://doi.org/10.1038/s41598-018-30487-6

Park, L., Uekawa, K., Garcia-Bonilla, L., Koizumi, K., Murphy, M., Pistik, R., Younkin, L., Younkin, S., Zhou, P., Carlson, G., Anrather, J., \& ladecola, C. (2017). Brain perivascular macrophages initiate the neurovascular dysfunction of Alzheimer abeta peptides. Circulation Research, 121(3), 258-269. https:// doi.org/10.1161/CIRCRESAHA.117.311054

Pugazhenthi, S., Qin, L., \& Reddy, P. H. (2017). Common neurodegenerative pathways in obesity, diabetes, and Alzheimer's disease. Biochimica et Biophysica Acta - Molecular Basis of Disease, 1863(5), 1037-1045. https://doi.org/10.1016/j.bbadis. 2016.04.017

Rivera-Escalera, F., Matousek, S. B., Ghosh, S., Olschowka, J. A., \& O'Banion, M. K. (2014). Interleukin-1beta mediated amyloid plaque clearance is independent of CCR2 signaling in the APP/ PS1 mouse model of Alzheimer's disease. Neurobiology Disease, 69, 124-133. https://doi.org/10.1016/j.nbd.2014.05.018

Sadahiro, M., Erickson, C., Lin, W. J., Shin, A. C., Razzoli, M., Jiang, C., Fargali, S., Gurney, A., Kelley, K. A., Buettner, C., Bartolomucci, A., \& Salton, S. R. (2015). Role of VGF-derived carboxyterminal peptides in energy balance and reproduction: analysis of "humanized" knockin mice expressing full-length or truncated VGF. Endocrinology, 156(5), 1724-1738. https://doi.org/ 10.1210/en.2014-1826

Sadahiro, M., Erickson, C., Lin, W. J., Shin, A. C., Razzoli, M., Jiang, C., Fargali, S., Gurney, A., Kelley, K. A., Buettner, C., Bartolomucci, A., \& Salton, S. R. (2014). A role for VGF in the hypothalamic arcuate and paraventricular nuclei in the control of energy homeostasis. Neuroscience, 265, 184-195. https:// doi.org/10.1016/j.neuroscience.2014.01.060

Sathe, G., Na, C. H., Renuse, S., Madugundu, A. K., Albert, M., Moghekar, A., \& Pandey, A. (2019). Quantitative proteomic profiling of cerebrospinal fluid to identify candidate biomarkers for Alzheimer's disease. Proteomics - Clinical Applications, 13(4), e1800105. https://doi.org/10.1002/prca.201800105

Thakker-Varia, S., Behnke, J., Doobin, D., Dalal, V., Thakkar, K. Khadim, F., Wilson, E., Palmieri, A., Antila, H., Rantamaki, T., \& Alder, J. (2014). VGF (TLQP-62)-induced neurogenesis targets early phase neural progenitor cells in the adult hippocampus and requires glutamate and BDNF signaling. Stem Cell Research, 12(3), 762-777. https://doi.org/10.1016/j.scr.2014. 03.005

Ueno, M., Chiba, Y., Matsumoto, K., Murakami, R., Fujihara, R., Kawauchi, M., Miyanaka, H., \& Nakagawa, T. (2016). Bloodbrain barrier damage in vascular dementia. Neuropathology, 36 (2), 115-124. https://doi.org/10.1111/neup. 12262

van Steenoven, I., Noli, B., Cocco, C., Ferri, G. L., Oeckl, P., Otto, M., Koel-Simmelink, M. J. A., Bridel, C., van der Flier, W. M., Lemstra, A. W., \& Teunissen, C. E. (2019). VGF peptides in cerebrospinal fluid of patients with dementia with Lewy bodies. International Journal of Molecular Science, 20(19), Article 4674. https://doi.org/10.3390/ijms20194674

\section{History}

Received March 1, 2021

Accepted March 31, 2021

Published online May 11, 2021

\section{Conflict of Interest}

The authors declare no conflict of interest.

\section{Editorial Note}

The acting editor was Peter Schönknecht.

\section{Funding}

Open access publication enabled by Otto von Guericke University Magdeburg

\section{ORCID}

Stefan Busse

(iD) https://doi.org/0000-0003-3354-0380

\section{Stefan Busse}

Department of Psychiatry

University of Magdeburg

Leipziger Str. 44

39120 Magdeburg

Germany

stefan.busse@med.ovgu.de 